\title{
Evaluation of Sealing Potential of Different Repair Materials in Furcation Perforations Using Dye Penetration: An In Vitro Study
}

\author{
Dennis Mohan ${ }^{1}$, Abhinav K Singh ${ }^{2}$, Feby Kuriakose ${ }^{3}$, Reema Malik ${ }^{4}$, Joseph Joy ${ }^{5}$, Dhanya John ${ }^{6}$
}

\begin{abstract}
Aim: The present study aimed to assess the sealing ability of various repair materials in furcation perforations using dye penetration.

Materials and methods: A total of 80 human first molars from mandible with unbroken enamel surfaces, which were extracted completely for periodontal reasons, were chosen. All extracted teeth were kept in a sterile solution up until further use. Endo-access bur was used to prepare endodontic access cavity, and a high-speed long shank round bur was used to create a planned perforation on the floor of pulp. All the specimens were randomly grouped (20 teeth in each group): Group I: control; group II: Pro-Root MTA; group III: resin-modified glass-ionomer cement (RM GIC); and group IV: Biodentin. After placement of repair materials in perforated furcation, the chamber of each tooth was accessed with $2 \%$ methylene blue dye. Later, a diamond disk was used to split the teeth buccolingually. A stereomicroscope (10X) was used to visualize the highest dye penetration.

Results: The least penetration was seen in teeth repaired by Biodentin group $(0.88 \pm 0.02)$, next by Pro-Root MTA group (1.24 \pm 0.13$)$ and resinmodified glass ionomer cement group ( $3.62 \pm 0.46$ ). A statistically significant difference was seen among different perforation repair materials by analysis of variance. A statistically significant difference ( $p$ value $<0.05$ ) was found between each group, excluding group II v/s group IV.

Conclusion: This study concluded that reduced dye penetration in perforation repair along the furcations and improved sealing ability are shown by biodentine group in comparison to Pro-Root MTA and resin-modified glass-ionomer cement group.

Clinical significance: Root perforations along the furcation that develop due to the endodontic procedures have a remarkable damaging effect on prognosis and frequently result in loss of secondary periodontal attachment, thus resulting in early loss of the involved tooth. Therefore, the selection of biocompatible repair material aids to enhance the treatment prognosis.
\end{abstract}

Keywords: Biodentine, Dye penetration, Perforation, Pro-Root mineral trioxide aggregate, Resin-modified glass-ionomer cement.

The Journal of Contemporary Dental Practice (2021): 10.5005/jp-journals-10024-2968

\section{INTRODUCTION}

Perforations in root may develop either due to iatrogenic reasons such as endodontic treatment or due to pathologic reasons such as caries and resorption. These root perforations complicate the response to treatment if not addressed. ${ }^{1}$

As per Ingle, an endodontic perforation is an opening created artificially in the tooth or its root by the clinician during root canal entry or due to a biological incident such as caries or pathological resorption, leading to a communication between periodontal tissue and the root canal. Apart from caries or resorptive defects, perforations of furcation or root are iatrogenic and are the main cause for root canal treatment failure. The second highest cause of endodontic failure is root perforations and accounts for $9.62 \%$ of all failed cases. The worst consequence of endodontic treatment is mostly a perforation of furcation, which is an opening along the mid curvature of root into the periodontal space. ${ }^{2}$

Conventionally, the root perforations are non-surgically treated with the use of materials that have the potential to mineralize, similar to closure of immature apices. The nonsurgical method of coronal treatment includes repair material placement immediately into the site of root perforation to evade the possible infection of the wound by bacteria. ${ }^{3}$

There are several materials with the potential to seal perforations. Some of these materials are glass ionomer cement, mineral trioxide aggregate (MTA), resin cements, resin-modified
${ }^{1}$ Department of Conservative Dentistry and Endodontics, Pariyaram Dental College, Pariyaram, Kannur, Kerala, India

${ }^{2}$ Department of Conservative Dentistry and Endodontics, Buddha Institute of Dental Sciences, Patna, Bihar, India

3,5 Department of Conservative Dentistry and Endodontics, Mar Baselios Dental College, Kothamangalam, Kerala, India

${ }^{4}$ Department of Conservative Dentistry and Endodontics, Maulana Azad Institute of Dental Sciences, Delhi, India

${ }^{6}$ Department of Conservative Dentistry and Endodontics, Annoor Dental College, Kerala, India

Corresponding Author: Dennis Mohan, Department of Conservative Dentistry and Endodontics, Pariyaram Dental College, Pariyaram, Kannur, Kerala, India, Phone: +91 9446441810, e-mail: drdennismohan@gmail.com

How to cite this article: Mohan D, Singh AK, Kuriakose F, et al. Evaluation of Sealing Potential of Different Repair Materials in Furcation Perforations Using Dye Penetration: An In Vitro Study. J Contemp Dent Pract 2021;22(1):80-83.

Source of support: Nil

Conflict of interest: None

glass ionomer cement, and zinc oxide eugenol cement (intermediate restorative material, super-ortho ethoxy benzoic acid). So far, several trials have shown that no material has fulfilled all the ideal requirements. ${ }^{4}$ 
The utilization of MTA has been recommended by most clinicians because of its fixing capacity, peripheral variation, and biocompatibility. Be that as it may, MTA displays the hindrances of expanded starting setting time, trouble in dealing with (contingent upon the water/powder proportion), and significant expense. Nonetheless, resin-modified glass ionomer cement demonstrated more microleakage and less fixing capacity in contrast to MTA cement. In order to beat these burdens, another material, biodentine, has been recently delivered in the market. It appears to have comparable physiochemical, mechanical, and natural properties to MTA yet shorter setting time with no aluminate or calcium sulfate in its composition. The major criteria of repair materials used for furcation perforation are its ability to seal, biocompatibility, and control of the flow of repair materials, as the root perforation is considered as an endless pit in which the escape of the repair material along the periodontal area is common. This further leads to severe periodontal tissue damage. ${ }^{5}$ Thus, this study was performed to assess the potential of Pro-Root MTA, resinmodified glass-ionomer cement, and biodentine repair materials to seal furcation perforations with the use of dye penetration method.

\section{Materials and Methods}

This in vitro study was performed in the Department of Conservative Dentistry and Endodontics, Pariyaram Dental College, Kerala, India. A total of 80 human mandibular first molars with unbroken enamel surfaces and nonfused, well-developed roots, which are not affected by resorption, fluorosis, absence of fracture and were extracted completely for periodontal reasons were chosen. All extracted teeth were stored in a sterile solution at room temperature until use.

Each specimen was cleaned of any tissue remnants on the roots and disinfected with $0.5 \%$ chloramine $T$ for 7 days, scaled by periodontal scalers, and subsequently stored in $0.9 \%$ normal saline solution. A high-speed air turbine handpiece with an endo-access bur was used to gain an initial entry and prepare endodontic access cavity, followed by use of Endo-z bur for extension of cavity laterally and to finish the cavity walls. The canal orifices were negotiated and temporary filling materials were placed in each canal orifice. Two layers of nail varnish were coated on each root's outer surface so as to avoid penetration of dye from minor dentine defects, open tubules, or lateral canals, with utmost attention to the furcation area. Intentional perforation was created on the pulpal floor by using a high-speed round bur no. $4(1.4 \times 1.4 \mathrm{~mm})$ with a long shank was positioned between the distal and the mesial orifices. Salinemoistened cotton was placed in tubes, and all the specimens were kept in tubes so as to reproduce the clinical condition.

\section{Repair of the Perforations}

A total of 80 specimens were randomly separated into four groups (20 teeth in each group)

\section{Group I: Control}

No material was used to repair the furcation.

\section{Group II: Pro-Root MTA}

The instructions given by the manufacturer were followed to mix $1 \mathrm{~g}$ of Pro-Root MTA (Dentsply Tulsa, Tulsa, OK, USA) with distilled water $(0.35 \mathrm{~mL})$ to yield a homogeneous paste. An Endogun (Medidenta Int. Inc., Woodside, NY, USA) was used to place ProRoot MTA material in the perforation, and Schilder pluggers
(Hu Friedy, Chicago, IL, USA) was used to compact the material. A saline-moistened cotton pellet was kept against the Pro-Root MTA in the pulp chamber.

\section{Group III: Resin-modified Glass-ionomer Cement}

A Vitremer primer was used to treat dentine for 30 seconds. Resin-modified glass ionomer cement (Vitremer) [3M ESPE, Dental Products, St. Paul, MN, USA] was applied using a 20-second lightinduced polymerization method before the application of glass ionomer cement. The instructions of manufacturer were followed to mix Vitremer powder and liquid. The perforation was packed with mixed cement, and endodontic plugger was used to compact the cement. A dental polymerization lamp "Curing light XL 3,000" (3M ESPE, Dental Products, St. Paul, MN, USA) was used for 40 seconds to polymerize the surface.

\section{Group IV: Biodentin}

As per the instructions of the manufacturer, Biodentine (Septodont, Saint-Maur-des-fosses Cedex, France) was mixed to obtain the most appropriate consistency. The material present within the capsule was taken, transferred to perforation site using a spatula, and amalgam pluggers were used to condense the material gently, and next 12 minutes were let for material to set.

\section{Preparation of Specimens for Analysis Using Dye Penetration Method}

The specimens from each group were kept in individual Petri dishes for 24 hours, and then methylene blue dye (2\%) was further added to access every specimen's pulp chamber in order to prepare the specimen for orthograde dye test. All specimens were kept for 48 hours. Once the specimens were removed from the dye, the specimens were subjected to a 40-minute thorough rinse under running water, and a polishing disk was used to remove varnish. A high-speed lathe with a diamond disk was used to section the teeth in a buccolingual direction. A stereomicroscope of magnification $10 \times$ was used to visualize the extent of dye penetration. Dye penetration was measured from the coronal level of the repair material to the apical end of the perforation by single investigator.

\section{Statistical Analysis}

The mean and standard deviation were calculated using the SPSS software (version 20.0). The comparisons within and between different perforation repair materials were measured using one-way analysis of variance (ANOVA). $p$ values $<0.05$ was considered statistically significant.

\section{Results}

Table 1 shows the mean and standard deviation of penetration of dye marginally among the study groups. The mean values for control group, Pro-Root MTA group, resin-modified glass ionomer cement group, and Biodentine group were $6.10 \pm 0.10,1.24 \pm 0.13$, $3.62 \pm 0.46$, and $0.88 \pm 0.02$, respectively.

Table 2 demonstrates the comparison of mean of penetration of dye marginally between the study groups. Among the perforation repair materials that were included, the least penetration was seen in teeth perforations repaired with Biodentine group $(0.88 \pm 0.02)$, next by Pro-Root MTA group (1.24 \pm 0.13$)$, and resin-modified glass ionomer cement group $(3.62 \pm 0.46)$. A statistically significantly different result was seen between the perforation repair materials as per analysis of variance. 
Table 1: Mean and standard deviation of marginal dye penetration between the study groups

\begin{tabular}{lll}
\hline Groups & $n$ & $\begin{array}{l}\text { Mean value } \pm \text { Std. } \\
\text { deviation }\end{array}$ \\
\hline Group I: control & 20 & $6.10 \pm 0.10$ \\
Group II: Pro-Root MTA & 20 & $1.24 \pm 0.13$ \\
$\begin{array}{l}\text { Group III: resin-modified } \\
\text { glass-ionomer cement }\end{array}$ & 20 & $3.62 \pm 0.46$ \\
Group IV: Biodentine & 20 & $0.88 \pm 0.02$ \\
\hline
\end{tabular}

Table 2: Comparison of mean marginal dye penetration between the study groups

\begin{tabular}{lllll}
\hline Groups & Mean $\pm S D$ & Fvalue & pvalue & Significance \\
\hline Group I: control & $6.10 \pm 0.10$ & 22.264 & 0.001 & HS \\
$\begin{array}{l}\text { Group II: Pro-Root } \\
\text { MTA }\end{array}$ & $1.24 \pm 0.13$ & & & \\
$\begin{array}{l}\text { Group III: resin- } \\
\text { modified glass- }\end{array}$ & $3.62 \pm 0.46$ & & & \\
ionomer cement & & & & \\
Group IV: biodentine & $0.88 \pm 0.02$ & & & \\
\hline
\end{tabular}

$p<0.05$, HS, highly significant

Table 3: Multiple comparison of mean difference between the study groups

\begin{tabular}{llcl}
\hline Group & Compared with & Mean difference (I-J) & Sig. \\
\hline Group I & Group II & 4.86 & 0.001 \\
& Group III & 2.48 & 0.001 \\
\multirow{4}{*}{ Group II } & Group IV & 5.52 & 0.001 \\
& Group I & -4.86 & 0.001 \\
& Group III & -2.38 & 0.04 \\
Group III & Group IV & 0.88 & 0.09 \\
& Group I & -2.48 & 0.001 \\
& Group II & 2.38 & 0.03 \\
Group IV & Group IV & 2.74 & 0.001 \\
& Group I & -5.22 & 0.001 \\
& Group II & -0.36 & 0.08 \\
& Group III & -2.74 & 0.001 \\
\hline
\end{tabular}

*Significant, $p<0.05$

Table 3 demonstrates the multiple comparisons between the different groups of perforation repair materials. Each group demonstrated a statistically significant difference ( $p$ value $<0.05$ ), excluding group II v/s group IV.

The inference of the present study indicates that the Biodentine group showed better sealing ability and less dye penetration in furcation perforation repair followed by Pro-Root MTA and resinmodified glass ionomer cement groups, respectively.

\section{Discussion}

Incorrect management of inadvertent root perforations results in treatment complications and affects the prognosis. It is vital to maintain the perforation site free from infections and restore the perforations immediately. In order to choose an appropriate repair material, the material's ability to seal and thus prevent expected extrusion into the furcation area should be measured. Also, the perforated site should be clearly visible in order to aid the repair procedure. $^{6}$
The extent of reaction of tissue to treatment of openings with various repair materials depends on some factors: the dimensions and area of openings, the ability of material to fix or cause cytotoxicity, the extent of damage to the periodontal tissue, the removal and destruction of bacteria, and the period lost before the damage is repaired. The openings along the coronal third which involves the furcation zone had high expectancy due to the proximity to gingival sulcus. The perfect estimate outcomes are seen when an opening is slightly far from the furcation zone or gingival sulcus and when the perforation gets sealed quickly. ${ }^{7}$

In this study, highest dye penetration was recorded in resin-modified glass ionomer cement group in comparison to Pro-Root MTA group and Biodentine group. This was recorded in RM-GIC group possibly due to the material's polymerization contraction. The bonding could get affected when the dentin gets contaminated with undue dampness, solvent, or presence of voids, thus complicating the treatment outcome. These findings are in accordance with a previous trial by Watts et al. ${ }^{8}$ in which significant changes were reported in similar RM-GIC properties in neutral and aqueous media.

The penetration of dye procedure has been in use in endodontics since long due to its advantage of performance and the associated challenges of other available methods. Nevertheless, there are many demerits, such as reduced size of the molecules of dye than bacteria, which do not evaluate the definite volume absorbed by the sample but just assess the deepest point the dye can reach. ${ }^{9}$ It is not easy to understand the definite depth the dye penetrates, as it relies on the arbitrary cutting of the tooth into two. Despite these disadvantages, Torabinejad et al. ${ }^{10}$ specified that a material which is capable of avoiding small molecules (dye) penetration would be able to avoid penetration of larger substances such as bacteria and its by-products.

In this trial, the least dye penetration was seen in Biodentine group, next by Pro-Root MTA and resin-modified glass ionomer cement group. However, the difference between Pro-Root MTA group and biodentine group was not statistically significant. As per the manufacturer, biodentin displays reduced porosity than Pro-Root MTA and has increased compressive strength during the period of first hour. A new study by Aggarwal et al. ${ }^{11}$ demonstrated lower push-out bond strength of MTA in the initial 24 hours period compared to Biodentine. The mechanical strength of the material enhances over time and attains greater than $200 \mathrm{MPa}$ at 24 hours, a value much beyond of that of the most glass ionomer cements.

Furthermore, Biodentin's compressive strength, modulus of elasticity, hardnes, $s$ and micro-mechanical anchorage to tooth structure is similar to that described for human dentine. Additionally, aluminates that lead to brittleness and weakening of the set material is not a chemical component. Also, Biodentin is nonresorbable, less soluble, hydrophilic, stable, and forms a tight seal. ${ }^{12-14}$ These findings are same as those obtained by Katge et al. ${ }^{15}$ and Sinkar et al. ${ }^{16}$ who reported increased sealing capability of Biodentin in perforations along furcation and Mir et al. ${ }^{17}$ who recorded significantly better sealing capability of Biodentin in comparison to bioactive bone cement and MTA. In the present study, control group demonstrated maximum dye penetration all through the cavities, therefore affirming that furcation fix materials were important to forestall microleakage.

In our study, no statistically significant difference was seen between Pro-Root MTA group and Biodentine group. The study 
by Main et al. ${ }^{18}$ assessed the rate of success of root perforation restorations with MTA and reported that MTA provides an efficient seal for perforations of root and exhibited the possibility of better prognosis of perforated teeth that would else be affected.

The limitations of present study are that the findings are stated in terms of penetration of dye with regard to the walls of perforation because perforation depth of the cavities is an uncontrolled variable reliant on the thickness of cementum and dentine of each tooth. Besides, these alterations in depth may be much greater in clinical conditions assuming that perforations are usually a consequence of extreme grinding of floor of pulp in order to trace orifices of calcified root canals. Other in vivo and clinical trials are necessary so as to explain the efficiency of the perforation repair materials.

\section{Conclusion}

This study concluded that reduced dye penetration in perforation repair along the furcations and improved sealing ability are shown by Biodentine group in comparison with Pro-Root MTA and resinmodified glass ionomer cement group.

\section{References}

1. Kakani AK, Veeramachaneni C, Majeti C, et al. A Review on perforation repair materials. J Clin Diagnos Res 2015;9(9):ZE09-ZE13.

2. Mohan TM, Sudha K, Shoba C, et al. Comparative evaluation of sealing ability of ProRoot MTA, biodentine and bone cement in the repair of furcation perforation - an in vitro study. Indian J Dent Adv 2019;10(4):176-180.

3. Bogaerts P. Treatment of root perforations with calcium hydroxide and Super EBA cement: a clinical report. Int Endod J 1997;30(3):210219. DOI: 10.1111/j.1365-2591.1997.tb00699.x.

4. El Tawil SB, El Dokkyl NA, El Hamid DA. Sealing ability of MTA versus portland cement in the repair of furcal perforations of primary molars: a dye extraction leakage model. J Am Sci 2011;7:1037-1043.

5. Komath M, Varma HK. Fully injectable calcium phosphate cement- a promise to dentistry. Indian J Dent Res 2004;15:89-95.
6. Daoudi MF. Microscopic management of endodontic procedural errors: perforation repair. Dent Update 2001;28(4):176-180. DOI: 10.12968/denu.2001.28.4.176.

7. Mangala TM, Pawar RR. Evaluating sealing ability of three different furcation perforation repair materials. JCR 2020;7(7):658-661.

8. Watts DC, Kisumbi B, Toworfe GK. Dimensional changes of resin/ ionomer restoratives in aqueous and neutral media. Dent Mater 2000;16(2):89-96. DOI: 10.1016/S0109-5641(99)00098-6.

9. Camps J, Pashley DH. Reliability of the dye penetration studies. J Endod 2003;29(9):592-594. DOI: 10.1097/00004770-20030900000012.

10. Torabinejad M, Rastegar AF, Kettering JD, et al. Bacterial leakage of mineral trioxide aggregate as a root-end filling material. J Endod 1995;21(3):109-112. DOI: 10.1016/S0099-2399(06)80433-4.

11. Aggarwal V, Miglani S, Kohli S, et al. Comparative evaluation of pushout bond strength of ProRoot MTA, biodentine, and MTA plus in furcation perforation repair. J Conservat Dentis 2013;16(5):462-465. DOI: 10.4103/0972-0707.117504.

12. Arora DV, Nikhil V, Sharma N, et al. Bioactive dentin replacement. IOSR-JDMS 2013;12(4):51-57. DOI: 10.9790/0853-1245157.

13. Bachoo IK, Seymour D, Brunton P. A biocompatible and bioactive replacement for dentine: is this a reality? the properties and uses of a novel calcium-based cement. Br Dent J 2013;214(2):E5. DOI: 10.1038/ sj.bdj.2013.57.

14. Butt N, Bali A, Talwar S, et al. Comparison of physical and mechanical properties of mineral trioxide aggregate and Biodentine. Indian J Dent Res 2014;25(6):692-697. DOI: 10.4103/0970-9290.152163.

15. Katge FA, Shivasharan PR, Patil D. Sealing ability of mineral trioxide aggregate Plus ${ }^{\mathrm{TM}}$ and Biodentin ${ }^{\mathrm{TM}}$ for repair of furcal perforation in primary molars: an in vitro study. Contemp Clin Dent 2016;7(4):487492. DOI: 10.4103/0976-237X.194100.

16. Sinkar R, Patil S, Jogad N, et al. Comparison of sealing ability of ProRoot MTA, RetroMTA, and Biodentine as furcation repair materials: an ultraviolet spectrophotometric analysis. J Conserv Dent 2015;18(6):445-448. DOI: 10.4103/0972-0707.168803.

17. Mir A, Misgar OH, Farooq R, et al. Comparison of sealing ability of biodentine, bioactive bone cement and MTA as furcation repair materials. IOSR J Dent Med Sci (IOSR-JDMS) 2017;16:82-86.

18. Main C, Mirzayan N, Shabahang S, et al. Repair of root perforations using mineral trioxide aggregate: a long-term study. J Endod 2004;30(2):80-83. DOI: 10.1097/00004770-200402000-00004. 